\title{
New data on the spider fauna (Arachnida, Aranei) of the Altai Territory
}

\author{
V.V. Sidorov, M.S. Galyuta \\ Altai State University \\ Lenina 61, Barnaul, 656049, Russia \\ E-mail: vladsidorov777@gmail.com
}

A checklist of 30 spider species recorded from the Tigirek State Nature Reserve (the Altai Territory) is provided, of which four species are recorded from the reserve for the first time and one is new to the Altai Mts. Also, the family Uloboridae was first recorded for the reserve.

Key words: Araneae; fauna; new records; spiders; Tigirek State Nature Reserve; Altai

\section{Introduction}

The Tigirek State Nature Reserve is situated in the northwestern part of the Altai Mts. Its territory lies at the altitude ranging from 500 to $2000 \mathrm{~m}$ a.s.l. Most of the reserve relief is middle-mountain, with dome-shaped peaks.

Special studies of the reserve's spider fauna began relatively recently. The first annotated checklist of spiders of the Tigirek Reserve was published by Trilikauskas and included 132 species (Volynkin et al., 2011). Later, the number of species was increased to 153 thanks to a series of publications by Azarkina \& Trilikauskas (2012, 2013a, b; Trilikauskas, 2014). The latest paper by Fomichev [2016] already contains information on 175 spider species recorded from the territory of the Tigirek Reserve. Nevertheless, the spider fauna of the reserve remains poorly inventoried.

\section{Methods}

This paper is based on the spider material collected by the senior author, A.S. Slepenkov and A.E. Naydenov in the period of 1-13 July, 2016. The spider species recorded from the Tigirek Reserve for the first time are marked with an asterisk ( $\left.{ }^{*}\right)$, and those that are new to the Altai Mts with two asterisks $\left({ }^{* *}\right)$. The studied material has been deposited in the collection of the Institute of Systematic and Ecology of Animals SB RAS, Novosibirsk, Russia (curator: G.N. Azarkina).

\section{Species List}

\section{Family AGELENIDAE}

Agelena labyrinthica (Clerck, 1758)

MATERIAL. c. 3 km NW of Tigirek Vil., Mayak Mt. (51 $\left.10^{\prime} \mathrm{N}, 83^{\circ} 00^{\prime} \mathrm{E}\right), 700-750 \mathrm{~m}, 01 . \mathrm{VII} .2016$ (Sidorov V.V.), $10^{\prime \prime}$.

DISTRIBUTION. Trans-Palaearctic nemoral range [Marusik et al., 2000].

Family ARANEIDAE

Aculepeira ceropegia (Walckenaer, 1802)

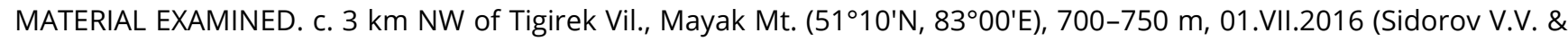
Slepenkov A.S.), 6\%; Tigirek Mt. Range, Razrabotnaya Mt. (5101'N, 8301'E), 1800-1960 m, 13.VII.2016 (Sidorov V.V.), 39.

DISTRIBUTION. Euro-Siberian polyzonal range [WSC, 2019].

Araniella displicata (Hentz, 1847)

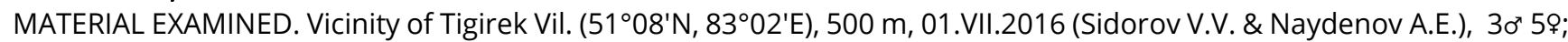

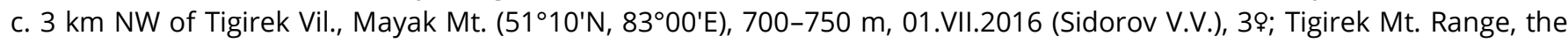

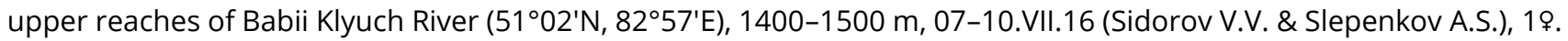

DISTRIBUTION. Circum-Holarctic boreo-nemoral range [Marusik et al., 2000]. 
Larinioides cornutus (Clerck, 1758)

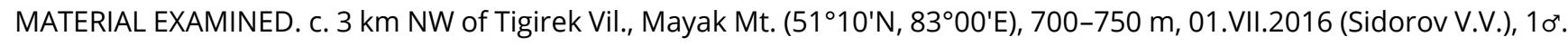
DISTRIBUTION. Circum-Holarctic polyzonal range [Marusik et al., 2000].

\section{Larinioides patagiatus (Clerck, 1758)}

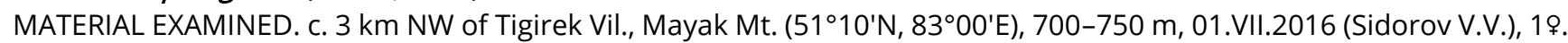
DISTRIBUTION. Circum-Holarctic polyzonal range [Marusik et al., 2000].

Mangora acalypha (Walckenaer, 1802)

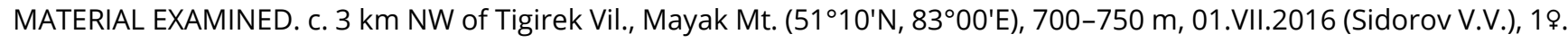
DISTRIBUTION. European-Altaian range [Azarkina \& Trilikauskas, 2012].

Singa nitidula C.L. Koch, 1844

MATERIAL EXAMINED. Vicinity of Tigirek Vil. (51ํㅇ' $\left.\mathrm{N}, 83^{\circ} 02^{\prime} \mathrm{E}\right), 500 \mathrm{~m}, 01 . \mathrm{VII.2016}$ (Sidorov V.V. \& Naydenov A.E.), 19.

DISTRIBUTION. Trans-Palaearctic (?) boreo-nemoral range [Marusik et al., 2000].

\section{Family CHEIRACANTHIIDAE}

\section{Cheiracanthium erraticum}

MATERIAL EXAMINED. Tigirek Mt. Range, the upper reaches of Babii Klyuch River (51을' $\left.\mathrm{N}, 82^{\circ} 57^{\prime} \mathrm{E}\right), 1400-1500 \mathrm{~m}, 07-$ 10.VII.16 (Sidorov V.V. \& Slepenkov A.S.), 19.

DISTRIBUTION. Trans-Palearctic polyzonal range [WSC, 2019].

*Cheiracanthium pennyi O. Pickard-Cambridge, 1873

MATERIAL EXAMINED. Tigirek Mt. Range, the upper reaches of Babii Klyuch River (51 $\left.{ }^{\circ} 02^{\prime} \mathrm{N}, 82^{\circ} 57^{\prime} \mathrm{E}\right), 1400-1500 \mathrm{~m}, 07-$ 10.VII.16 (Sidorov V.V. \& Slepenkov A.S.), 10 '.

DISTRIBUTION. Euro-Siberian polyzonal range [WSC, 2019].

*Cheiracanthium punctorium (Villers, 1789)

MATERIAL EXAMINED. c. 3 km NW of Tigirek Vil., Mayak Mt. (51 $\left.{ }^{\circ} 10^{\prime} \mathrm{N}, 83^{\circ} 00^{\prime} \mathrm{E}\right), 700-750 \mathrm{~m}, 01$. VII.2016 (Sidorov V.V.), $10^{\prime}$. DISTRIBUTION. West Palaearcric temperate range [WSC, 2019].

\section{Family CLUBIONIDAE}

Clubiona pseudosaxatilis Mikhailov, 1992

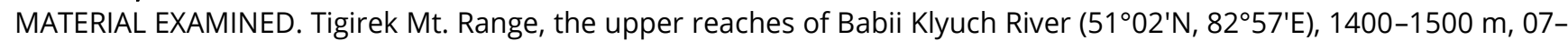
10.VII.16 (Sidorov V.V. \& Slepenkov A.S.), 10\%; Tigirek Mt. Range, Razrabotnaya Mt. (51ํำ' $\left.\mathrm{N}, 83^{\circ} 01^{\prime} \mathrm{E}\right), 1800-1960 \mathrm{~m}$, 13.VII.2016 (Slepenkov A.S.), 7 9.

DISTRIBUTION. West Mongolian range [Mikhailov, 1992].

\section{Family GNAPHOSIDAE}

Drassodes cupreus (Blackwall, 1834)

MATERIAL EXAMINED. Tigirek Mt. Range, the upper reaches of Babii Klyuch River (51을' $\left.\mathrm{N}, 82^{\circ} 57^{\prime} \mathrm{E}\right), 1400-1500 \mathrm{~m}, 07-$ 10.VII.16 (Sidorov V.V. \& Slepenkov A.S.), 19.

DISTRIBUTION. Trans-Palaearctic boreo-nemoral range [Marusik et al., 2000].

Gnaphosa banini Marusik et Koponen, 2001

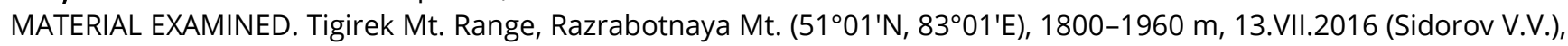
59.

DISTRIBUTION. Altai-Mongolian mountain range [Azarkina \& Trilikauskas, 2013a].

Parasyrisca volynkini(Fomichev, 2016)

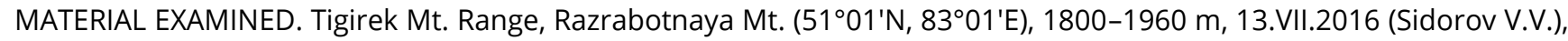
29, 29 subad.

DISTRIBUTION. The type locality only [Fomichev, 2016; Fomichev, Marusik \& Sidorov, 2018].

Family LYCOSIDAE

Acantholycosa cf. altaiensis Marusik, Azarkina et Koponen, 2004 
MATERIAL EXAMINED. Tigirek Mt. Range, the upper reaches of Babii Klyuch River $\left(51^{\circ} 02^{\prime} \mathrm{N}, 82^{\circ} 57^{\prime} \mathrm{E}\right), 1400-1500 \mathrm{~m}, 07-$

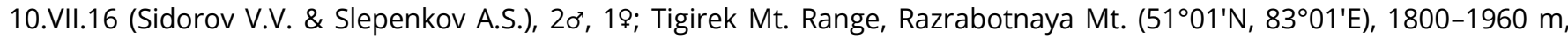
13.VII.2016 (Slepenkov A.S.), 20', 19.

DISTRIBUTION. Endemic of North-Western Altai [Marusik et al., 2004].

COMMENTS. Collected specimens resembles Acantholycosa altaiensis, but slightly different in palpal characters. They most likely belong to a new species and needs further attention, which is beyond scope of this paper.

Alopecosa aculeata (Clerck, 1758)

MATERIAL EXAMINED. Tigirek Mt. Range, the upper reaches of Babii Klyuch River (5102'N, 8257'E), 1400-1500 m, 0710.VII.16 (Sidorov V.V. \& Slepenkov A.S.), 4 \% .

DISTRIBUTION. Circum-Holarctic polyzonal range [Marusik et al., 2000].

\section{Pardosa agrestis/plumipes}

MATERIAL EXAMINED. Vicinity of Tigirek Vil. (51ํㅇ' $\left.\mathrm{N}, 83^{\circ} 02^{\prime} \mathrm{E}\right), 500 \mathrm{~m}, 01 . \mathrm{VII} .2016$ (Sidorov V.V. \& Naydenov A.E.), 29. DISTRIBUTION. West Palaerctic - West Siberian nemoral range [Marusik et al., 1996].

COMMENTS. Males of two species, Pardosa agrestis (Westring, 1861) and P. plumipes (Thorell, 1875), can be easily distinguished by formation of first legs; males of $P$. plumipes have long and dense hairs on tibiae, metatarsi and tarsi of first legs, while in $P$. agrestis this character is absent. Females of these two species are hardly distinguishable from each other. The only way to identify females is collect it with males (Azarkina, personal data).

Pardosa lugubris (Walckenaer, 1802)

MATERIAL EXAMINED. Vicinity of Tigirek Vil. (51 $\left.{ }^{\circ} 08^{\prime} \mathrm{N}, 83^{\circ} 02^{\prime} \mathrm{E}\right), 500 \mathrm{~m}, 01 . \mathrm{VII} .2016$ (Sidorov V.V. \& Naydenov A.E.), 19; Tigirek Mt. Range, the upper reaches of Babii Klyuch River (51 $\left.{ }^{\circ} 02^{\prime} \mathrm{N}, 82^{\circ} 57^{\prime} \mathrm{E}\right), 1400-1500$ m, 07-10.VII.16 (Sidorov V.V. \& Slepenkov A.S.), 1 \%.

DISTRIBUTION. West Palaearctic temperate range [WSC, 2019].

\section{Family PHILODROMIDAE}

Philodromus cespitum (Walckenaer, 1802)

MATERIAL EXAMINED. Vicinity of Tigirek Vil. (51 $\left.{ }^{\circ} 08^{\prime} \mathrm{N}, 83^{\circ} 02^{\prime} \mathrm{E}\right), 500 \mathrm{~m}, 01 . \mathrm{VII} .2016$ (Sidorov V.V. \& Naydenov A.E.), $10^{\prime \prime}, 29 ;$ C. 4 km NW of Tigirek Vil., (51 $\left.{ }^{\circ} 09^{\prime} \mathrm{N}, 82^{\circ} 59^{\prime} \mathrm{E}\right), 600-700 \mathrm{~m}, 03 . \mathrm{VII} .2016$ (Sidorov V.V.), $10^{\circ}$.

DISTRIBUTION. Circum-Holarctic polyzonal range [Marusik et al., 2000].

Philodromus emarginatus (Schrank,1803)

MATERIAL EXAMINED. Vicinity of Tigirek Vil. (51 $\left.{ }^{\circ} 08^{\prime} \mathrm{N}, 83^{\circ} 02^{\prime} \mathrm{E}\right), 500 \mathrm{~m}, 01 . \mathrm{VII.2016}$ (Sidorov V.V. \& Naydenov A.E.), $10^{\star}$. DISTRIBUTION. Trans-Palearctic nemoral range [Marusik et al., 2000].

\section{Family SALTICIDAE}

Calositticus floricola (C. L. Koch, 1837)

MATERIAL EXAMINED. Tigirek Mt. Range, the upper reaches of Babii Klyuch River $\left(51^{\circ} 02^{\prime} \mathrm{N}, 82^{\circ} 57{ }^{\prime} \mathrm{E}\right), 1400-1500 \mathrm{~m}, 07-$ 10.VII.16 (Sidorov V.V. \& Slepenkov A.S.), 6 \%.

DISTRIBUTION. Trans-Palaearctic boreo-nemoral range [Marusik et al., 2000].

\section{Family SPARASSIDAE}

Micrommata virescens (Clerk, 1757)

MATERIAL EXAMINED. Vicinity of Tigirek Vil. (51ํㅇ' $\left.\mathrm{N}, 83^{\circ} 02^{\prime} \mathrm{E}\right), 500 \mathrm{~m}, 01 . \mathrm{VII.2016}$ (Sidorov V.V. \& Naydenov A.E.), $10^{\star}$. DISTRIBUTION. Trans-Palearctic nemoral range [Marusik et al., 2000].

Family THERIDIIDAE

Parasteatoda tepidariorum (C.L. Koch, 1841)

MATERIAL EXAMINED. c. 4 km NW of Tigirek Vil., (51ํํ'N, 8259'E), 600-700 m, 03.VII.2016 (Sidorov V.V.), 1 \%.

DISTRIBUTION. Cosmopolitan range [Marusik et al., 2000].

Phylloneta impressa (L. Koch, 1881)

MATERIAL EXAMINED. c. 3 km NW of Tigirek Vil., Mayak Mt. (51¹0'N, 8300'E), 700-750 m, 01.VII.2016 (Sidorov V.V.), 29. DISTRIBUTION. Trans-Palearctic - North-West Nearctic polyzonal range [Marusik et al., 2000]. 
Lysiteles maius Ono, 1979

MATERIAL EXAMINED. Tigirek Mt. Range, Razrabotnaya Mt. (51 $\left.01^{\circ} \mathrm{N}, 83^{\circ} 01^{\prime} \mathrm{E}\right), 1800-1960$ m, 13.VII.2016 (Sidorov V.V.), $10^{7}$.

DISTRIBUTION. Altaian-Nepalo-Manchurian disjunctive nemoral range [Azarkina \& Trilikauskas, 2013b].

**Xysticus lanio C. L. Koch, 1835 (Fig. 1)

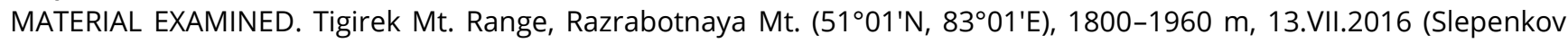
A.S.), $10^{7}$.

DISTRIBUTION. West Palaearctic temperate range [WSC, 2019].

\section{Xysticus ulmi(Hahn, 1831)}

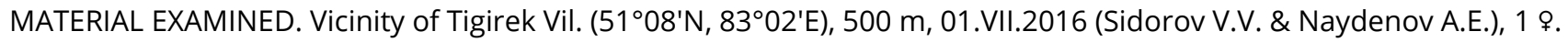
DISTRIBUTION. West-Central Palaearctic temperate range [Esyunin et al., 2013].

Xysticus obscurus (Collett, 1877)

MATERIAL EXAMINED. Tigirek Mt. Range, the upper reaches of Babii Klyuch River (51을 $\left.\mathrm{N}, 82^{\circ} 57^{\prime} \mathrm{E}\right), 1400-1500 \mathrm{~m}, 07-$ 10.VII.16 (Sidorov V.V. \& Slepenkov A.S.), 19.

DISTRIBUTION. Circum-Holarctic boreo-alpine range [Marusik et al., 2000].

Psammitis bonneti(Denis, 1938)

MATERIAL EXAMINED. Tigirek Mt. Range, the upper reaches of Babii Klyuch River (51을 $\left.\mathrm{N}, 82^{\circ} 57^{\prime} \mathrm{E}\right), 1400-1500 \mathrm{~m}, 07-$ 10.VII.16 (Sidorov V.V. \& Slepenkov A.S.), 2 ..

DISTRIBUTION. European-Baikalian disjunctive boreoalpine range [Marusik et al., 2000].

\section{Family ULOBORIDAE}

*Uloborus walckenaerius Latreille, 1806

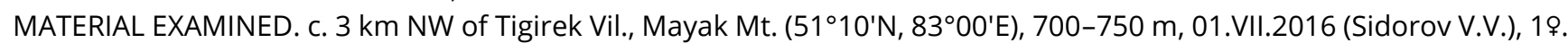
DISTRIBUTION. Trans-Palearctic nemoral range [Marusik et al., 2000].

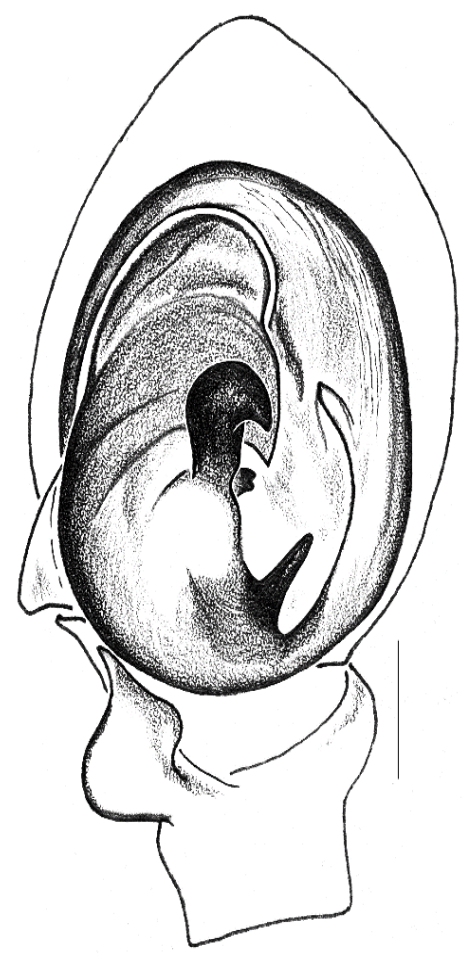

Fig. 1. Male palp of Xysticus lanio C. L. Koch, 1835 (ventral view). Scale bar $-0.1 \mathrm{~mm}$.

\section{Results}

The spider fauna of the Tigirek State Nature Reserve is complemented by three additional species and now accounts for 180 species in 98 genera and 23 families. Even so, the araneofauna of the reserve seems to consist of more than 240 species (Fomichev, 2016). 


\section{Acknowledgements}

We are grateful to P.V. Golyakov, T.M. Krugova, R.V. Yakovlev, A.A. Shmakov (all from Barnaul, Russia) for their help in the organization of the field trip to the reserve. Also, we wish to thank A.S. Slepenkov (Sovetsky, Russia), A.E. Naydenov (Barnaul, Russia) for their great help with collecting the studied spider material, to G.N. Azarkina (Novosibirsk, Russia) for checking our identifications and helping with drawing. D.V. Logunov (Manchster, UK) kindly edited the English of the final draft.

\section{References}

Azarkina, G.N., Trilikauskas, L.A. (2012). New data on spider fauna (Aranei) of the Russian Altai, part I: families Agelenidae, Araneidae, Clubionidae, Corinnidae, Dictynidae and Eresidae. Euroasian Entomological Journal, 11(3), $199-208$.

Azarkina, G.N., Trilikauskas, L.A. (2013a). New data on spider fauna (Aranei) of the Russian Altai, part II: families Gnaphosidae, Hahniidae, Linyphiidae, Liocranidae and Lycosidae. Euroasian Entomological Journal, 12(1), 51-67.

Azarkina, G.N., Trilikauskas, L.A. (2013b). New data on spider fauna (Aranei) of the Russian Altai, part III: families Mimetidae, Miturgidae, Oxyopidae, Philodromidae, Pholcidae, Pisauridae, Salticidae, Sparassidae, Tetragnathidae, Theridiidae, Thomisidae, Titanoecidae, Uloboridae and Zoridae. Euroasian Entomological Journal, 12(3), 243-254.

Davydov, E.A., Botchkareva, E.N., Chernykh, D.V. (2011). [Natural conditions of the Tigirek Strict Nature Reserve]. Trudy Tigirekskogo Zapovednika. Barnaul, 4, 7-19 (in Russian).

Esyunin, S.L., Ermakov, A.I., Mikhailov, Yu.E. (2013). Remarks on the Ural spider fauna (Arachnida: Aranei), 14. On the spider fauna of the Kytlym plexus of mountains (the North Urals). Arthropoda Selecta, 22(1), 75-82.

Fomichev, A. A. (2016). New data on the spiders (Arachnida: Aranei) from Altai Territory, Russia. Arthropoda Selecta, 25(1), 119-126.

Fomichev, A. A., Marusik, Y. M. \& Sidorov, V. V. (2018). A survey of East Palaearctic Gnaphosidae (Aranei). 9. New data on the Parasyrisca potanini-group from Central Asia. Arthropoda Selecta, 27(2), 155-168.

Mikhailov, K.G. (1992). The spider genus Clubiona Latreille, 1804 (Arachnida Aranei Clubionidae) in the USSR fauna: a critical review with taxonomic remarks. Arthropoda Selecta, 1(3), 3-34.

Trilikauskas, L.A. (2014). [On some seasonal aspects of spiders and harvestmen population (Arachnida: Aranei, Opiliones) in larch forests of the Tigirekski Reserve (North-Western Altai). Tomsk State University Journal of Biology, 28(4), 123-135 (in Russian).

Volynkin, A.V., Trilikauskas, L.A., Baghirov, R.T-O., Burmistrov, M.V., Byvaltsev, A.M., Vasilenko, S.V., Vishnevskaya, M.S., Danilov, Yu.N., Dudko, A.Yu., Dudko, R.Yu., Knyshov, A.A., Kosova, O.V., Kostrov, D.V., Krugova, T.M., Kuznetsova, R.O., Kuzmenkin, D.V., Legalov, A.A., Lvovsky, A.L., Namyatova, A.A., Nedoshivina, S.V., Perunov, Yu.E., Reschnikov, A.V., Sinev, S.Yu., Solovarov, V.V., Tyumaseva, Z.I., Udalov, I.A., Ustyuzhanin, P.Ya., Filimonov, R.V., Tshernyshev, S.E., Tshesnokova, S.V., Sheikin, S.D., Shcherbakov, M.V., Yanygina, L.V. (2011). [Invertebrates of the Tigirek Nature Reserve (an annotated checklist), Biota of the Tigirek Natural Reserve]. Trudy Tigirekskogo Zapovednika. Barnaul, 4, 165-226 (in Russian). WSC 2019. World Spider Catalog, Version 20.0. Natural History Museum Bern. Available from: http://wsc.nmbe.ch/ accessed on 14 June, 2019.

\section{Citation:}

Sidorov,V.V., Galyuta, M.S. (2019). New data on the spider fauna (Arachnida, Aranei) of the Altai Territory. Acta Biologica Sibirica, 5 (2), 35-39.

Submitted: 07.04.2019. Accepted: 23.06.2019

cross ref http://dx.doi.org/10.14258/abs.v5.i2.5930

(C) 2019 by the authors. Submitted for possible open access publication under the terms and conditions of the

Creative Commons Attribution (CC BY) license (http://creativecommons.org/licenses/by/4.0/). 